\title{
Aspectos clínicos de um paciente portador de Síndrome de CHARGE: estudo de caso
}

\author{
Clinical aspects of a patient with CHARGE Syndrome: case report
}

Aspectos clínicos de un paciente con Síndrome CHARGE: reporte de caso

Nathalie Bartelega Dominguetti ${ }^{1 *}$, Carolina Ponciano Gomes de Freitas ${ }^{1}$, Paula Ester Mendes Barbosa Fernandes ${ }^{1}$, Luiza Almeida Brandão ${ }^{2}$, Fernanda Guimarães Avelar ${ }^{1}$, Camilla de Paiva Silva Ferreira $^{1}$, Fátima Lúcia Guedes Silva ${ }^{1}$.

\section{RESUMO}

Objetivo: Discutir os aspectos e o manejo da Síndrome de CHARGE, uma condição genética rara, causada por uma mutação esporádica dominante no gene $\mathrm{CHD7}$, a partir do acompanhamento ambulatorial de um portador da condição. Detalhamento de Caso: BFCSB, sexo feminino, sete anos, apresenta disfunção oftalmológica e auditiva, disfunção no nervo óptico, atraso no desenvolvimento e crescimento, malformação cardiovascular, fenda orofacial já submetida à cirurgia e malformações auditivas. Além disso, possui dificuldade na modulação do afeto e otites supurativas recorrentes. Possui história familiar negativa para Síndrome de CHARGE. Apresenta adequado suporte familiar e assistência multidisciplinar. No caso da paciente do estudo, a suspeita da Síndrome de CHARGE foi baseada em critérios maiores e menores e confirmada através das mutações heterozigóticas no gene CHD7. O acompanhamento por uma equipe multidisciplinar foi fundamental para o desenvolvimento da paciente, bem como tratamento precoce de situações clínicas encontradas. Considerações Finais: A paciente apresenta a maioria das alterações clínicas previstas na literatura para a Síndrome de CHARGE. O diagnóstico precoce e a assistência em saúde por uma equipe multidisciplinar permitiram bons resultados.

Palavras-chave: Pediatria, Síndrome CHARGE, Cardiopatias congênitas.

\section{ABSTRACT}

Objective: To discuss the aspects and management of CHARGE Syndrome, a rare genetic condition, caused by a sporadic dominant mutation in the $\mathrm{CHD7}$ gene, based on the outpatient monitoring of a person with the condition. Case Details: BFCSB, female, seven years old, has ophthalmic and auditory dysfunction, optic nerve dysfunction, development and growth delay, cardiovascular malformation, orofacial cleft already submitted to surgery and hearing malformations. In addition, she has difficulty in modulating affection and recurrent suppurative ear infections. She has a negative family history for CHARGE Syndrome. She presents adequate family support and multidisciplinary assistance. In the case of the study patient, the suspicion of CHARGE syndrome was based on major and minor criteria and confirmed through heterozygous mutations in the CHD7 gene. A follow-up by a multidisciplinary team was essential for the patient's development, as well as early treatment of clinical situations encountered. Final Considerations: The patient presents most of the clinical alterations foreseen in the literature for CHARGE Syndrome. Early diagnosis and health care by a multidisciplinary team allowed good results.

Key words: Pediatrics, CHARGE Syndrome, Heart defects congenital.

${ }^{1}$ Universidade Federal de Ouro Preto (UFOP), Ouro Preto - MG. *E-mail: nathaliedominguetti@gmail.com

2 Instituto de Previdência dos Servidores do Estado de Minas Gerais (IPSEMG), Belo Horizonte - MG. 


\section{RESUMEN}

Objetivo: Discutir los aspectos y el manejo del Síndrome CHARGE, una condición genética rara, causada por una mutación dominante esporádica en el gen $\mathrm{CHD7}$, basado en el seguimiento ambulatorio de una persona con la condición. Detalles del Caso: BFCSB, mujer, siete años, tiene disfunción oftálmica y auditiva, disfunción del nervio óptico, retraso en el desarrollo y crecimiento, malformación cardiovascular, hendidura orofacial ya sometida a cirugía y malformaciones auditivas. Además, tiene dificultad para modular el afecto y las infecciones supurativas recurrentes del oído. Tiene antecedentes familiares negativos de síndrome CHARGE. Presenta un adecuado apoyo familiar y asistencia multidisciplinar. En el caso del paciente del estudio, la sospecha de síndrome CHARGE se basó en criterios mayores y menores y se confirmó mediante mutaciones heterocigotas en el gen CHD7. El seguimiento por parte de un equipo multidisciplinar fue fundamental para el desarrollo del paciente, así como el tratamiento temprano de las situaciones clínicas encontradas. Consideraciones Finales: La paciente presenta la mayoría de las alteraciones clínicas previstas en la literatura para el Síndrome CHARGE. El diagnóstico precoz y la atención sanitaria por parte de un equipo multidisciplinario permitieron buenos resultados.

Palabras clave: Pediatría, Síndrome CHARGE, Cardiopatías congénitas.

\section{INTRODUÇÃO}

A Síndrome de CHARGE é uma condição genética rara, causada por uma mutação dominante e esporádica no gene CHD7, que regula a transcrição de vários genes específicos de tecidos (LALANI SR, et al., 2006; HSU P, et al., 2014). A doença é caracterizada por uma série de malformações, que formando um acrônimo, dão o nome à síndrome. São elas: coloboma de íris, cardiopatia congênita, atresia de coanas, retardo no crescimento e desenvolvimento, anomalias genitais e no pavilhão auricular/surdez (FORMIGA CKMR, et al., 2014; VILLEGAS LC, et al., 2010).

Além das características clássicas anteriormente descritas, outras potenciais manifestações a nível otológico, rinológico, oral e faringolaríngeo estão incluídas no espetro da síndrome de CHARGE. A confirmação genética pode ser feita na maioria dos pacientes por detecção de mutações heterozigóticas no gene CHD7. Uma anamnese completa e exame físico bem realizado são cruciais, uma vez que o diagnóstico, seguido de intervenção precoce, pode melhora a qualidade de vida do paciente (SANLAVILLE D e VERLOES A, 2007).

O processo ocorre por um erro na migração das células da crista neural, e consequentemente há uma interação anormal com o prosencéfalo, mesencéfalo e rombencéfalo, gerando parte das anomalias citadas (PIETRO C, et al., 2010; SANLAVILLE D e VERLOES A, 2007). Nos colobomas oculares típicos, por exemplo, a alteração ocorre pelo errático fechamento da fissura embrionária da vesícula óptica, que se inicia no nervo óptico e progride até a margem inferonasal ou inferior da pupila, alteração que ocorre em consequência da mutação descrita (TEIXEIRA C, et al., 2001).

A prevalência da doença é estimada em 1 caso para cada 8.500-15.000 nascidos vivos e a falha no desenvolvimento inicia no primeiro mês de gestação (SANCHEZ N, et al. 2019). Em geral, a mortalidade é elevada nos primeiros anos de vida em decorrência das diversas malformações congênitas e necessidade de numerosas cirurgias para correção. Malformações cardíacas complexas e de vias aéreas, associada à dificuldade alimentar, deficiência imune e limitação anestésica aumentam o risco cirúrgico. Após os primeiros anos a mortalidade ainda é alta, devido à infecções e hospitalizações frequentes.

Já na adolescência e idade adulta a mortalidade reduz devido à menor necessidade de cirurgias, entretanto ainda é maior em relação à população geral devido a infecções, aspiração de conteúdo gástrico e apneia obstrutiva. Os pacientes com a síndrome costumam necessitar continuamente de cuidados. Contudo, mesmo com essas diversas complicações o paciente pode ter expectativa de vida normal (HEFNER MA e FASSI E, 2017). 
Quanto às alterações genéticas, apesar de a maioria dos autores concordarem com a esporadicidade da mutação, segundo Tellier AL (1996), há um maior número de casos de síndrome de CHARGE entre crianças filhas de pais com idade maior que 33 anos de idade, em comparação com crianças filhas de pais mais jovens. Essa associação não foi demonstrada em relação à idade da mãe.

O diagnóstico da síndrome de CHARGE é realizado primariamente a partir dos aspectos clínicos, divididos em critérios maiores e menores. Segundo Verloes A (2005), os maiores são o coloboma ocular, a atresia de coanas e as anomalias do pavilhão auricular. Já os critérios menores são: disfunção rombencefálica (anomalias do tronco cerebral e nervos cranianos), disfunção hipotálamo-hipofisário, malformações da orelha interna/externa, malformação de órgãos mediastinais (coração, esôfago) e atraso cognitivo.

É importante ressaltar que o diagnóstico e a realização de intervenções precoces nas principais alterações da síndrome permitem melhores desfechos. O déficit sensorial auditivo, por exemplo, causa prejuízos na aquisição e desenvolvimento da linguagem oral, prejudicando o aprendizado, e possui com uma alternativa terapêutica a cirurgia de implante coclear (CARDOSO CC, et al., 2013).

O estudo em questão tem como objetivo discutir os principais aspectos clínicos da síndrome de CHARGE, a abordagem diagnóstica e acompanhamento desses pacientes, a partir da experiência do caso descrito e de revisão da literatura, para contribuir com a ampliação do campo de conhecimento desta condição rara e pouco manejada na rotina médica atual. Trata-se de uma descrição de caso clínico de caráter descritivo e explicativo, e a pesquisa bibliográfica foi realizada a partir de busca eletrônica nas bases de dados Pubmed e Scielo, a partir de descritores como síndrome de CHARGE, cardiopatias congênitas e abordagem de malformações.

\section{DETALHAMENTO DO CASO}

BFCSB, sexo feminino, sete anos, residente da região central de MG. É acompanhada no ambulatório desde o primeiro ano de vida, quando foram iniciados os cuidados da puericultura. Acerca da história gestacional, a gravidez foi desejada, a mãe da paciente teve uma gestação anterior com filho saudável, e realizou pré-natal adequadamente, a única intercorrência foi um episódio de infecção urinária. A criança nasceu de parto cesárea, $2920 \mathrm{~g}, 47 \mathrm{~cm}$ de estatura, $32 \mathrm{~cm}$ de perímetro cefálico, Apgar de 9 no $1^{\circ}$ minuto e 10 no 5 o minuto. Já na primeira consulta com pediatra, com um mês de vida, foi diagnosticado coloboma de íris e lábio leporino com fenda palatina.

No primeiro ano de vida, apresentou atraso no desenvolvimento neuropsicomotor, infecções de ouvido recorrentes e hipoacusia. Por esse motivo a criança foi encaminhada ao otorrinolaringologista e à psicopedagogia. A suspeita de Síndrome de CHARGE foi levantada ainda no primeiro ano de vida da paciente, a partir das características clínicas apresentas. Sendo assim, a paciente foi encaminhada para 0 geneticista que ratificou o diagnóstico da síndrome.

O quadro clínico da paciente contempla dois critérios maiores e quatro critérios menores da síndrome. Sobre os critérios maiores, a paciente apresenta características de disfunção oftalmológica relacionada a erros de refração, associada ao coloboma de íris. A suspeita clínica foi levantada durante a consulta, quando a criança se aproximou demasiadamente de objetos para poder observá-los. Além disso, apresenta diminuição da acuidade auditiva, associada à hipoplasia de canais semicirculares.

Somado a isso, a paciente apresentava os seguintes critérios menores: atraso no desenvolvimento (a paciente foi incluída no ensino regular, porém não obteve êxito em acompanhar a velocidade de aprendizagem dos demais alunos de classe), malformação cardiovascular, atraso no crescimento, fenda orofacial já submetida à cirurgia, malformações do conduto auditivo, e disfunção no nervo óptico (fato constatado pelo oftalmologista que acompanha a criança). A hipoplasia genital não foi avaliada no caso.

Além desses critérios, foi verificado que a criança apresentava dificuldade na modulação do afeto, visto que durante a consulta demonstrou variação extrema de suas expressões emocionais, alternando momentos de colaboração com momentos de irritação intensa, sem nenhum estímulo significativo prévio. Também foi relatado pela mãe a presença de otites supurativas recorrentes. No que se refere à história familiar, não foram verificados outros casos de Síndrome de CHARGE. O único detalhe observado em relação aos fatores genéticos predisponentes foi a idade paterna, que, na época do nascimento da paciente, ultrapassava os 33 anos. 
Em relação às características psicossociais do caso, a paciente apresenta adequado suporte familiar, visto que a mãe dedica tempo significativo para educá-la e levá-la aos serviços de saúde que acompanham o caso. Nesse sentido, vale ressaltar que a criança é acompanhada pelo pediatra, geneticista, otorrinolaringologista, oftalmologista, cardiologista e psicopedagoga. Além disso, conseguiu obter bom relacionamento interpessoal, principalmente com os colegas e professores da escola.

\section{DISCUSSÃO}

O diagnóstico da paciente foi realizado conforme previsto pelos protocolos da doença. Houve a suspeita clínica baseada em critérios maiores e menores e posteriormente mutações heterozigóticas no gene CHD7 foram detectadas pelo geneticista. Tais mutações alteram a transcrição de vários genes específicos de tecidos, o que gerou as alterações clínicas no quadro da paciente (HSU P, et al., 2014). Como observado nos estudos anteriormente citados, essas mutações são reconhecidas, atualmente, como esporádicas, mas observa-se que na história familiar da paciente seu pai apresenta idade maior que 33 anos, corroborando com uma vertente que tenta associar a idade paterna avançada com a síndrome em questão (TELLIER AL, et al., 1996).

As alterações apresentadas pela paciente estão em acordo com a epidemiologia esperada para os portadores da Síndrome de CHARGE. As deformidades visuais, apresentadas no caso, estão presentes na maioria dos pacientes, assim como as malformações cardíacas, como a Tetralogia de Fallot, que também aparecem com grande frequência (HSU P, et al., 2014).

O crescimento e desenvolvimento da paciente também ocorreram conforme o esperado para a Síndrome. Esses pacientes costumam nascer com peso e altura adequados para a idade, mas frequentemente crescem pouco durante a infância. Em geral, também estão comprometidas as funções cognitivas e de linguagem. Já a atresia de coanas, achado muito comum, não foi encontrada no caso. Além disso, a hipoplasia genital, que pode ocorrer por deficiência nos níveis dos hormônios sexuais, não foi avaliada na paciente (HSU P, et al., 2014).

A perda auditiva condutora e/ou neurossensorial pode ocorrer na Síndrome de CHARGE. Já na orelha interna são comuns a ausência de canais laterais semicirculares, displasia vestibular e malformações cocleares (COSTA JR, et al., 2020; DE GEUS C M, et al.,2017). Raramente podem ser apresentadas outras disfunções endócrinas, em nervos cranianos, e imunodeficiência. Quanto à última, tem-se exemplificado no caso em questão o relato materno de infecções supurativas de ouvido recorrentes. Esta situação pode estar relacionada a malformações da tuba auditiva, e também com disfunções da imunidade humoral. (VERLOES A, 2005; ROJAS M e WALKER L, 2012)

Quando houver a suspeita da síndrome em questão, a criança deve ser referenciada a um geneticista, para avaliação do diagnóstico, história familiar e opções de teste genético (SANCHEZ N, et al., 2019). Um dos sequenciamentos usados é o de Sanger, que detecta mutações na maioria dos casos. Diversas alterações no sequenciamento podem ser encontradas, como deleções, inserções e mutações pontuais (HSU P, et al., 2014). De acordo com o estudo de Aramaki M (2016), nas crianças com o diagnóstico clínico da síndrome de CHARGE, as mutações no gene CHD7 podem ser identificadas em $71 \%$ dos casos.

Existe um grande espectro de diagnósticos diferenciais para as alterações observadas na síndrome de CHARGE, por esse motivo, de acordo com o artigo publicado por Sousa PC (2017), acredita-se que estejam subestimados os dados atuais sobre incidência e prevalência da síndrome. As manifestações auditivas, oftálmicas, cardiovasculares, orofaciais, dos tratos genital e urinário e alterações de desenvolvimento neuropsicomotor e comportamentais possibilitam a elaboração de diversas hipóteses diagnósticas, como transtorno obsessivo-compulsivo, transtorno de déficit de atenção, síndrome de Tourette e autismo, mesmo porque crianças com a síndrome frequentemente desenvolvem essas alterações (HARTSHORNE T, et al., 2009).

Por esse motivo, torna-se necessária a exclusão cuidadosa dessas alternativas diagnósticas e a referência dos casos sugestivos para a avaliação e sequenciamento genético, com o objetivo de identificar a mutação que define a doença. 
Uma observação importante é quanto ao aconselhamento genético. A maioria das mutações no gene CHD7 acontece de forma que o risco da descendência de progenitores saudáveis (tendo já um filho com síndrome de CHARGE) é de, apenas, 2 a 3\%. Todavia, pelo fato de se tratar de uma mutação autossômica dominante, o risco na descendência gerada por portadores desta síndrome é de $50 \%$, em casos nos quais ocorre transmissão hereditária, as manifestações fenotípicas são, geralmente, atípicas (SOUSA PC, et al., 2017). Porém, como citado anteriormente, essas mutações são em sua maioria esporádicas.

A abordagem diagnóstica, intervenções precoces e acompanhamento longitudinal são necessários para que se obtenha melhores desfechos no desenvolvimento psicomotor e neurológico dos portadores da síndrome (TREVISI P, et al., 2016). Por esse motivo é necessário prontamente reconhecer as características clínicas do quadro. Como demonstrado no estudo de Cardoso CC, et al. (2013), o déficit sensorial auditivo, mesmo que leve, pode ter consequências no desenvolvimento da linguagem oral das crianças, e consequentemente no seu aprendizado e desempenho acadêmico.

Seguramente os pacientes com a síndrome CHARGE diferem um dos outros, mas existem desafios comuns. Um destes desafios é processar informações por meio de um sistema multissensorial distorcido. Eles podem alcançar coerência na experiência sensorial se conseguirem superar as limitações neurossensoriais, incluindo campo visual, controle postural e eficiência motora (BROWN D, 2005). Esses indivíduos tendem a gastar mais energia do que os demais que não apresentam a síndrome, para acompanhar as atividades que presenciam (SOURIAU J, et al., 2015).

Crianças com CHARGE precisam de auxílio para acessar eventos do mundo e das relações interpessoais. Sem a assistência adequada, elas podem não alcançar todo seu potencial de desenvolvimento. Estes indivíduos necessitam de uma rede de apoio competente em sua rotina. Essas crianças devem ser acompanhadas precocemente por uma equipe de atuação interdisciplinar, tanto para correção ou melhoria de parte das anomalias, quanto para o estímulo das funções cognitivas e psicomotoras (SOURIAU J, et al., 2005). A abordagem dos pacientes portadores de Síndrome de CHARGE deve incluir a interpretação dos resultados obtidos em estudos e suas principais implicações, sendo também relevante a comparação dos achados do caso clínico descrito com a literatura, e avaliar as limitações de cada estudo.

Conclui-se, portanto, que o caso da paciente em questão apresenta a maioria das alterações clínicas previstas na literatura para a Síndrome de CHARGE, o que favoreceu o diagnóstico e manejo precoces da condição. A partir disso, a criança obteve bons resultados com melhora da acuidade auditiva e resolução cirúrgica precoce da fenda orofacial, o que promoveu melhora significativa na qualidade de vida da criança. Ademais, a paciente possui acompanhamento adequado por uma equipe interdisciplinar, de forma que se beneficia amplamente da assistência em saúde, o que também proporciona uma abordagem mais adequada de cada individualidade que a criança apresenta. Sendo assim, é perceptível a importância do conhecimento dos profissionais da área da saúde a respeito dessa afecção, mesmo que rara, para que sejam capazes de realizar um diagnóstico precoce e assistir o paciente de forma eficiente, já que isso é essencial para melhoria e potencialização do desenvolvimento neuropsicomotor destes indivíduos.

\section{REFERÊNCIAS}

1. ARAMAKI M, et al. Phenotypic spectrum of CHARGE syndrome with CHD7 mutations. The Journal of Pediatrics, 2016; 148(3): 410-414.

2. BROWN D. CHARGE syndrome "behaviors": challenges or adaptations? American Journal of Medical Genetics part A, 2005; $133 A(3): 268-72$.

3. CARDOSO CC, et al. Cochlear Implants in Children Diagnosed with CHARGE Syndrome. International Archives Of Otorhinolaryngology, 2013; 17:424-428.

4. COSTA JR, et al. Bilateral Congenital Semicircular Canal Malformation and Hearing Loss - case report. Birth and Growth Medical Journal, 2020; 29(1): 36-42.

5. DE GEUS CM, et al. Guidelines in CHARGE syndrome and the missing link: Cranial imaging. American journal of medical genetics. Seminars in medical genetics, 2017; 175(4): 450-464.

6. FORMIGA CKMR, et al. Síndrome de Charge: relato de caso. Portal Regional da BVS: Biblioteca virtual em saúde, $2014 ; 13(75): 32-36$.

7. HARTSHORNE T, et al. Sleep disturbances in CHARGE syndrome: types and relationships with behavior and caregiver well-being. Developmental Medicine \& Child Neurology, 2009; 51: 143-150. 
8. HSU P, et al. CHARGE syndrome: A review. Journal of Paediatrics and Child Health 50. 2014; 504-511.

9. HEFNER MA, FASSI E. Genetic counseling in CHARGE syndrome: Diagnostic evaluation through follow up. American Journal of Medical Genetics Part C: Seminars in Medical Genetics, 2017; 175(4), 407-416.

10. LALANI SR, et al. Spectrum of CHD7 Mutations in 110 Individuals with CHARGE Syndrome and Genotype-Phenotype Correlation. The American Journal of Human Genetics, 2006; 78(2): 303-314.

11. PIETRO CJL, et al. El síndrome CHARGE. ArchArgentPediatr, 2010;108(1): e9-e12 / e9.

12. ROJAS M, WALKER L. Malformaciones Congénitas: Aspectos Generales y Genéticos. International Journal of Morphology, 2012; 30(4): 1256-1265.

13. SANCHEZ N, et al. Espectro fenotípico de Síndrome de CHARGE neonatal. Revista chilena de pediatria, 2019; 90(5): 533-538.

14. SANLAVILLE D, VERLOES A. CHARGE Syndrome: an update. European Journal of Human Genetics, 2007; 15: 389399.

15. SOURIAU J, et al. CHARGE syndrome: developmental and behavioural data. American Journal of Medical Genetics Part A, 2005; 133A(3): 278-81

16. SOUSA PC, et al. Síndrome de Charge - Dois casos clínicos com diferentes manifestações Otorrinolaringológicas. Acta Otorrinolaringol. Gallega, 2017; 10(1): 104-110..

17. TEIXEIRA C, et al. Associação de Charge e Cataratas Congénitas A Importância da Oftalmologia Pediátrica no Diagnóstico, Acta Oftalmológica, 2001; 11; 31-35.

18. TELLIER AL, et al. Increased paternal age in CHARGE association. Clinical Genetics, 1996; 50(6): 548-50.

19. TREVISI P, et al. Outcomes of long-term audiological rehabilitation in charge syndrome. Acta Otorhinolaryngologicaltalica, 2016; 36(3):206-14.

20. VERLOES A. Updated diagnostic criteria for CHARGE syndrome: a proposal. American Journal of Medical Genetics part A, 2005; 133A: 306-308.

21. VILLEGAS LC, et al. Síndrome CHARGE: reporte de caso y revisión de la literatura. latreia, 2010; 23(4-S): S-75. 